\title{
Approximate analytical method for finding eigenvalues of Sturm-Liouville problem with generalized boundary condition of the third kind
}

\author{
V. D. Lukyanov ${ }^{1}$, D. A. Bulekbaev ${ }^{2}$, A. V. Morozov², L. V. Nosova ${ }^{2}$ \\ ${ }^{1}$ Joint-Stock Company “Avangard”, Kondrat'evsky, 72, St. Petersburg, 195271, Russia \\ ${ }^{2}$ Mozhaisky Military Space Academy, Zhdanovskaya, 13, St. Petersburg, 197198, Russia \\ lukyanovvd@rambler.ru, atiman@mail.ru, alex.morozof@gmail.com,lvn1201@gmail.com
}

PACS 02.60.Lj, 47.61.Fg, 62.25.-g

DOI 10.17586/2220-8054-2020-11-3-275-284

\begin{abstract}
The Sturm-Liouville problem is solved for a linear differential second-order equation with generalized boundary conditions of the third kind Generalized boundary conditions consist of a linear combination of the boundary values of a function and its derivative. The coefficients of the linear combination are polynomials of the boundary problem eigenvalue. A method of approximate analytical calculation of boundary problem eigenvalues is proposed The calculation error of an eigenvalue is estimated.
\end{abstract}

Keywords: Sturm-Liouville problem, boundary conditions of the third kind, eigenfunctions, eigenvalues, approximation.

Received: 21 June 2020

\section{Introduction}

Advances of nanotechnologies make it possible to design and create microelectromechanical systems (MEMS) and nanoelectromechanical systems (NEMS). These systems find use as primary converters in miniature sensors of physical quantities [1].

Characteristic dimensions of used systems elements: hundreds of nanometers for MEMS with operating frequencies of sensors up to $10 \mathrm{gHz}$ and tens of nanometers for NEMS with operating frequencies of sensors up to tens of $\mathrm{gHz}[2,3]$.

High-quality electromechanical resonators are used as primary converters to provide sensor sensitivities and measurement accuracy of physical quantities. Various aspects of these devices research, technological developments and their various applications are presented in the reviews [4-12].

The simplest resonators in MEMS and NEMS are one-dimensional distributed elastic structures (strings, rods, beams loaded with sensitive elements - masses). Technologies for making such resonators are presented, for example, in $[13,14]$.

The mathematical model of time harmonic elastic oscillations of resonators is the Sturm-Liouville boundary problem. The Sturm-Liouville problem is a boundary problem on a segment for an ordinary linear homogeneous differential equation with homogeneous boundary conditions at the ends of the segment [15].

To describe the motion of an MEMS or NEMS element, a generalized boundary condition of the third kind is used, containing eigenvalues [15-17]. A generalized boundary condition is a linear combination of the sought function and its first-order derivative, wherein the coefficients of the linear combination are polynomials with respect to the sought eigenvalue of the problem. The mathematical theory such of problems is built in the works [18, 19], where a generalization of the classical Sturm-Liouville problem theory is given [20,21].

\section{Statement of the Sturm-Liouville problem with generalized boundary conditions of the third kind}

We consider the Sturm-Liouville boundary problem for a linear homogeneous ordinary second-order differential equation

$$
y^{\prime \prime}=-\mu y,
$$

where $y=y(x)$ at $-1 \leq x \leq 1, \mu$ is an eigenvalue of the problem, $\mu>0$. Taking into account the positivity $\mu$ we will accept $\mu=\lambda^{2}$, where $\lambda>0$.

As boundary conditions for the differential equation, we have generalized homogeneous conditions of the third kind [15-17]:

$$
\begin{gathered}
\alpha_{1}(\mu) y(1)+\beta_{1}(\mu) y^{\prime}(1)=0, \\
\alpha_{2}(\mu) y(-1)+\beta_{2}(\mu) y^{\prime}(-1)=0 .
\end{gathered}
$$

Multipliers $\alpha_{k}=\alpha_{k}(\mu)$ and $\beta_{k}=\beta_{k}(\mu)(k=1,2)$ are polynomials with respect to $\mu$. Polynomials coefficients are parameters of the problem. Parameters depend on characteristics of physical models in application problems. If the 
total number of such parameters under conditions (2) and (3) is equal to the number $S$, then we will call the boundary problem $S$-parametric. We will denote problem parameters by $\gamma_{1}, \gamma_{2}, \ldots \gamma_{S}$.

If the homogeneous boundary problem (1)-(3) has a non-zero solution $y_{0}(x)$ at a value $\mu=\mu_{0}$, then the value $\mu_{0}$ is called an eigenvalue, and the corresponding solution $y_{0}(x)$ is called an eigenfunction of the boundary problem.

\section{Sturm-Liouville problem eigenvalues calculation algorithm}

To solve the Sturm-Liouville problem means to find all the problem eigenvalues and eigenfunctions.

Eigenvalues are analytically calculated for the simplest boundary conditions of the first and second kind. In cases of boundary conditions of the third kind, eigenvalues are calculated as the roots of a transcendental equation lacking an exact analytical solution. Therefore, various approximate methods are used to find eigenvalues. These methods produce only an asymptotic estimate of an eigenvalue without discussing the accuracy of the result obtained, which is important in applied research.

The proposed new method makes it possible to calculate a set of boundary problem eigenvalues and to evaluate effectively the accuracy of their calculation.

Consider the problem of finding all eigenvalues and eigenfunctions of the Sturm-Liouville problem (1)-(3).

To solve the problem, we find the general solution of the differential equation (1)

$$
y=C_{1} \cos \lambda x+C_{2} \sin \lambda x,
$$

where $C_{1}$ and $C_{2}$ depend on the value of $\lambda$.

Satisfying the boundary conditions (2) and (3), we obtain a homogeneous system of linear algebraic equations for finding quantities $C_{1}$ and $C_{2}$ in the general solution (4):

$$
\left\{\begin{array}{l}
C_{1} p_{1}^{-}(\lambda)+C_{2} q_{1}^{+}(\lambda)=0, \\
C_{1} p_{2}^{+}(\lambda)+C_{2} q_{2}^{-}(\lambda)=0,
\end{array}\right.
$$

where

$$
\begin{gathered}
p_{k}^{ \pm}(\lambda)=\alpha_{k}\left(\lambda^{2}\right) \cos \lambda \pm \lambda \beta_{k}\left(\lambda^{2}\right) \sin \lambda, \\
q_{k}^{ \pm}(\lambda)= \pm \alpha_{k}\left(\lambda^{2}\right) \sin \lambda+\lambda \beta_{k}\left(\lambda^{2}\right) \cos \lambda
\end{gathered}
$$

at $k=1,2$. We take upper or lower symbol in formulas for $p_{k}^{ \pm}(\lambda)$ and $q_{k}^{ \pm}(\lambda)$ simultaneously.

The principal determinant of the homogeneous system of algebraic equations (5) is in the form of

$$
\Delta\left(\lambda, \gamma_{1}, \gamma_{2}, \ldots, \gamma_{S}\right)=p_{1}^{-}(\lambda) q_{2}^{-}(\lambda)-p_{2}^{+}(\lambda) q_{1}^{+}(\lambda) .
$$

The arguments of the determinant are a sought value $\lambda$ and parameters $\gamma_{1}, \gamma_{2}, \ldots, \gamma_{S}$ included in the conditions (2) and (3).

In order to find a non-zero solution to the homogeneous system of linear algebraic equations (5), we will require that the principal determinant of this system to be zero:

$$
\Delta\left(\lambda, \gamma_{1}, \gamma_{2}, \ldots, \gamma_{S}\right)=0 .
$$

Equation (7) is called a characteristic equation. To find an eigenvalues $\mu$ of the Sturm-Liouville problem, we look for the positive roots $\lambda$ of characteristic equation (7), and then look for the eigenvalue $\mu=\lambda^{2}>0$.

As can be seen from equality (6), the principal determinant is the sum of the components consisting of the works of the polynomial and trigonometric functions that depend on a sought $\lambda$ value. Thus, equation (7) is transcendental and does not allow an analytic solution of the form of

$$
\lambda=\varphi\left(\gamma_{1}, \gamma_{2}, \ldots, \gamma_{S}\right),
$$

which would allow to calculate an eigenvalue from known problem parameter values.

The proposed approximate method provides an approximate representation of the view (8) and estimates the accuracy of this representation.

Let us give an algorithm of the proposed method of obtaining an approximate solution of the characteristic equation.

In the first step we will make a table of function values $\lambda=\varphi\left(\gamma_{1}, \gamma_{2}, \ldots, \gamma_{S}\right)$ on some set of variable $\gamma_{1}, \gamma_{2}, \ldots, \gamma_{S}$ values. To do this, we record the determinant $\Delta\left(\lambda, \gamma_{1}, \gamma_{2}, \ldots, \gamma_{S}\right)$ as a linear combination of trigonometric functions with multipliers representing some polynomials with respect to $\lambda$ value. Coefficients of these polynomials are calculated through parameter $\gamma_{1}, \gamma_{2}, \ldots, \gamma_{S}$ values.

The dependence of the determinant on each parameter is not more than quadratic, since the problem parameters are coefficients of the polynomials and enter in the polynomials linearly, and the polynomials themselves are only multiplied according to formula (6). 
Using this circumstance, we solve equation (7) with respect to some arbitrarily chosen parameter $\gamma_{i}$ with the $i$ number. We get a functional dependence of the form of

$$
\gamma_{i}=\varphi_{i}\left(\lambda, \gamma_{1}, \gamma_{2}, \ldots, \gamma_{i-1}, \gamma_{i+1}, \ldots, \gamma_{S}\right)
$$

We now arbitrarily set argument values of the function $\varphi_{i}$ and calculate the value $\gamma_{i}$. For example, we can select arguments values of the function $\varphi_{i}$ on a uniform grid of the $\lambda, \gamma_{1}, \gamma_{2}, \ldots, \gamma_{i-1}, \gamma_{i+1}, \ldots, \gamma_{S}$ arguments. Values of the parameter $\gamma_{i}$ are then calculated. If necessary, if the values obtained for the parameter $\gamma_{i}$ are not sufficient, the relation (9) can be recorded with another selection of the parameter on the left hand side of the equality (9). We can now choose additional values for the $\gamma_{i}$ parameter. As a result, we create a table of function $\varphi_{i}$ values.

In the second step of solving characteristic equation we have a sufficient number of function $\varphi_{i}$ values. We find an approximating function $\Gamma_{i}=\Phi_{i}\left(\lambda, \gamma_{1}, \gamma_{2}, \ldots, \gamma_{i-1}, \gamma_{i+1}, \ldots, \gamma_{S}\right)$ using the obtained set of the function values $\varphi_{i}$ given by formula (9). We will select the approximation function $\Phi_{i}$ so that it has an inverse function $\Phi_{i}^{-1}$ with respect to the variable $\lambda$ at fixed values of the $\gamma_{2}, \ldots, \gamma_{i-1}, \gamma_{i+1}, \ldots, \gamma_{S}$ arguments:

$$
\lambda=\Phi_{i}^{-1}\left(\gamma_{1}, \gamma_{2}, \ldots, \gamma_{i-1}, \Gamma_{i}, \gamma_{i+1}, \ldots, \gamma_{S}\right)
$$

In order to calculate the approximate value of the sought $\lambda_{*}$ value, we set in this formula $\Gamma_{i}=\gamma_{i}$ :

$$
\lambda_{*}=\Phi_{i}^{-1}\left(\gamma_{1}, \gamma_{2}, \ldots, \gamma_{i-1}, \gamma_{i}, \gamma_{i+1}, \ldots, \gamma_{S}\right) .
$$

Then we calculate our eigenvalue $\mu_{*}=\lambda_{*}^{2}$.

As an example of the implementation of the method, we consider the problem of finding eigen frequencies of a longitudinally oscillating rod loaded with masses at the ends.

\section{Statement of eigen frequencies problem for a longitudinally oscillating rod loaded at the ends with masses}

We have an elastic uniform rod: the rod length is $2 l$, the constant cross-sectional area is $F$, the rod material has Young's modulus $E$, linear density is $\rho$. Body 1 with mass $M_{1}$ and body 2 with mass $M_{2}$ are fixed at the rod ends. The placement of rod (R) and bodies 1 and 2 in the coordinate system is shown in Fig. 1.

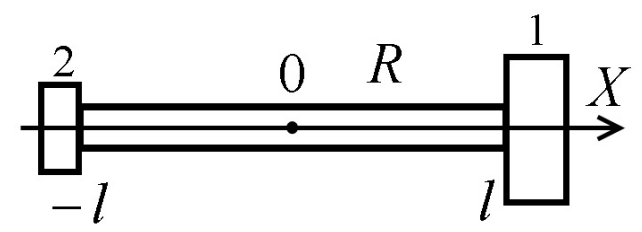

FIG. 1. Longitudinally oscillating rod (R) loaded at the ends with bodies 1 and 2. Choice of the coordinate system is shown

Small longitudinal displacements $U=U(X, t)$ of rod cross section with coordinate $X$ from equilibrium position at moment of time $t$ satisfy to the equation

$$
\rho F \frac{\partial^{2} U(X, t)}{\partial t^{2}}=E F \frac{\partial^{2} U(X, t)}{\partial X^{2}}
$$

at $-l<X<l$.

Boundary conditions describe masses oscillations under the action of the elastic rod:

we have on the right end of the $\operatorname{rod}$ if $X=l$

we have on the left end of the $\operatorname{rod}$ if $X=-l$

$$
M_{1} \frac{\partial^{2} U(l, t)}{\partial t^{2}}=-E F \frac{\partial U(l, t)}{\partial X},
$$

$$
M_{2} \frac{\partial^{2} U(-l, t)}{\partial t^{2}}=E F \frac{\partial U(-l, t)}{\partial X} .
$$

In order to find eigen frequencies of rod oscillations it is considered that dependence of longitudinal displacement of rod cross-section on time is harmonic with circular frequency $\omega: U(X, t)=Y(X) e^{-i \omega t}, Y=Y(X)$ is the amplitude of longitudinal displacement of rod cross-section at the point with coordinate $X$.

Let us pass in equations (11)-(13) to dimensionless values. We introduce the dimensionless coordinate $x=X / l$, then $-1 \leq x \leq 1$, and introduce the dimensionless amplitude of the longitudinal displacement of rod cross-section $y(x)=Y(X) / l$. 
We introduce also dimensionless quantities: dimensionless eigenvalue $\mu=\rho \omega^{2} l^{2} / E$, dimensionless eigen frequency $\lambda=\sqrt{\mu}$, and two dimensionless parameters of the problem: $\gamma_{1}=M_{1} / M_{0}$ and $\gamma_{2}=M_{2} / M_{1}$, where the mass $M_{0}$ is equal to half the mass of the rod: $M_{0}=\rho F l$. Note that problem parameters have the properties: $\gamma_{1}>0$, $\gamma_{2} \geq 0$.

Taking into account the introduced dimensionless quantities problem (11)-(13) takes the form of boundary problem (1)-(3)

$$
y^{\prime \prime}=-\lambda^{2} y
$$

at $-1 \leq x \leq 1$, with edge conditions of type (2) and (3) where $\alpha_{1}\left(\lambda^{2}\right)=\gamma_{1} \lambda^{2}, \alpha_{2}\left(\lambda^{2}\right)=\gamma_{1} \gamma_{2} \lambda^{2}$, here $\beta_{k}=$ $\beta_{k}\left(\lambda^{2}\right)=(-1)^{k}$ at $k=1,2$ :

$$
\begin{gathered}
\gamma_{1} \lambda^{2} y(1)-y^{\prime}(1)=0, \\
\gamma_{1} \gamma_{2} \lambda^{2} y(-1)+y^{\prime}(-1)=0 .
\end{gathered}
$$

We have a two-parametric boundary problem with parameters $\gamma_{1}$ and $\gamma_{2}$.

A simpler single-parameter problem is discussed in [22], when the masses attached to the rod ends are the same: $M_{1}=M_{2}$ or $\gamma_{2}=1$.

The general solution of equation (14) has the form (4). We obtain from the boundary conditions (15) and (16) a system of linear algebraic equations of the form of (5), where

$$
p_{k}^{ \pm}(\lambda)=\alpha_{k}\left(\lambda^{2}\right) \cos \lambda \pm \lambda \sin \lambda, \quad q_{k}^{ \pm}(\lambda)= \pm \alpha_{k}\left(\lambda^{2}\right) \sin \lambda \mp \lambda \cos \lambda
$$

at $k=1,2$.

The characteristic equation (7) for finding non-zero eigen frequencies is converted to the form of

$$
\gamma_{1}^{2} \gamma_{2} \lambda^{2} \sin 2 \lambda-\gamma_{1}\left(1+\gamma_{2}\right) \lambda \cos 2 \lambda-\sin 2 \lambda=0
$$

The obtained equation defines the implicit dependence of the dimensionless frequency $\lambda$ on the problem parameters $\gamma_{1}$ and $\gamma_{2}$. As shown in item 3 and seen from equation (17), it is not possible to obtain an analytical solution of the characteristic equation with respect to a sought value $\lambda$.

The proposed method of solving the characteristic equation makes it possible to obtain an approximate analytical $\lambda=\lambda\left(\gamma_{1}, \gamma_{2}\right)$ dependence.

Note that the characteristic equation (17) was obtained in 6.2 [23] in solving the problem of a substance diffusion through a permeable wall. The equation was solved graphically for the particular $\gamma_{2}=1$ case, when this equation is converted to the form of $\cot 2 \lambda=\left(\gamma_{1}^{2} \lambda^{2}-1\right) / 2 \gamma_{1} \lambda$. The graphs of functions from the right and left hand sides of the equation were drawn at a fixed value of the $\gamma_{1}$ parameter. The sought frequency values $\lambda$ were obtained as abscissas of the intersections points of these graphs.

As a result, in [23] there is a table of values of five first sought eigen frequency values $\lambda_{s}$ for three parameter $\gamma_{1}$ values. It is difficult to use the obtained results in practice: first, the number of frequencies found is insufficient for the qualitative numerical simulation of a physical problem, second, modeling may require a different parameter value $\gamma_{1}$ than the table value, third, the case $\gamma_{2} \neq 1$ will be interesting.

\section{Approximate solution of characteristic equation for the problem of longitudinal oscillations of elastic rod loaded with masses}

In computer simulation of practical problems it is convenient to have an approximate analytical solution of the characteristic equation for all roots of this equation and at any values of problem parameters.

Approximate methods used to solve equations of type (17) give analytical estimates for eigenvalues sufficient for large eigenvalues [24]. Obtaining approximate formulas for the first eigenvalues near zero causes difficulties. Estimates of the accuracy of approximate formulas for eigenvalues near zero are not usually considered. The proposed method of solving the characteristic equation allows for solve these problems.

\subsection{Finding dependencies of eigen frequency $\lambda$ on Sturm-Liouville problem parameters $\gamma_{1}$ and $\gamma_{2}$ in tabular and graphical view}

Let's take advantage of the fact that the characteristic equation (17) cannot be solved in elementary functions relative to the eigen frequency $\lambda$, but can be resolved relative to the parameters $\gamma_{1}$ or $\gamma_{2}$.

Consider the values $\lambda$ and $\gamma_{2}$ in the characteristic equation as independent variables $\left(0<\lambda<+\infty, \gamma_{2} \geq 0\right)$, and consider the parameter $\gamma_{1}>0$ as dependent variable. The left hand side of equation (17) is a quadratic function relative to the $\gamma_{1}$ variable. We find the dependence of the value $\gamma_{1}$ on the variables $\lambda$ and $\gamma_{2}$, if we calculate the roots of quadratic equation (17):

$$
\gamma_{1}^{ \pm}=\gamma_{1}^{ \pm}\left(\lambda, \gamma_{2}\right)=\frac{1}{2 \gamma_{2} \lambda^{2}}\left(W\left(\lambda, \gamma_{2}\right) \pm \sqrt{W^{2}\left(\lambda, \gamma_{2}\right)+4 \gamma_{2} \lambda^{2}}\right)
$$


where the function $W\left(\lambda, \gamma_{2}\right)=\left(1+\gamma_{2}\right) \lambda \cot 2 \lambda$ is used. In formula (18), either the upper or lower signs are taken at the same time.

Denote the functions $\gamma_{1}^{ \pm}=\gamma_{1}^{ \pm}\left(\lambda, \gamma_{2}\right)$ by $\gamma_{1 n}^{ \pm}=\gamma_{1 n}^{ \pm}\left(\lambda, \gamma_{2}\right)$ if argument $\lambda$ belongs to the interval $\left(\nu_{n-1}, \nu_{n}\right]$ with the number $n$ :

$$
\gamma_{1}^{ \pm}\left(\lambda, \gamma_{2}\right)=\gamma_{1 n}^{ \pm}\left(\lambda, \gamma_{2}\right) \text { at } \lambda \in\left(\nu_{n-1}, \nu_{n}\right]
$$

\subsection{Properties of $\gamma_{1}^{ \pm}\left(\lambda, \gamma_{2}\right)$ functions}

1. Functions $\gamma_{1}^{ \pm}\left(\lambda, \gamma_{2}\right)$ are defined for all $\lambda>0$ but values $\nu_{n}= \pm \pi n / 2$ at $n=1,2, \ldots$ Direct substitution of values $\lambda \neq \nu_{n}$ into equation (17) proves that relations (17) and (18) are equivalent.

2. Functions $\gamma_{1}^{ \pm}\left(\lambda, \gamma_{2}\right)$ are even relative to the $\lambda$ variable, since the function $W\left(\lambda, \gamma_{2}\right)$ in equality (18) is even, and the degrees of $\lambda$ are even.

3. Functions $\gamma_{1 n}^{ \pm}\left(\lambda, \gamma_{2}\right)$ are continuous on each $\left(\nu_{n-1}, \nu_{n}\right]$ interval.

4. Functions $\gamma_{1 n}^{ \pm}\left(\lambda, \gamma_{2}\right)$ are of constant signs: $\gamma_{1 n}^{+}\left(\lambda, \gamma_{2}\right) \geq 0, \gamma_{1 n}^{-}\left(\lambda, \gamma_{2}\right) \leq 0$. Taking into account the positivity of possible parameter $\gamma_{1}$ values we will study further only the functions $\gamma_{1 n}^{+}\left(\lambda, \gamma_{2}\right)$.

5. We have the equality $\gamma_{1 n}^{+}\left(\nu_{n+1}, \gamma_{2}\right)=0$ and the limit value $\gamma_{1 n}^{+}\left(\lambda, \gamma_{2}\right) \rightarrow+\infty$ as $\lambda \rightarrow \nu_{n}+0$.

6. The graphs of functions $\gamma_{1}^{+}(\lambda)=\gamma_{1 n}^{+}\left(\lambda, \gamma_{2}^{)}\right.$at $n=1,2,3$ are shown in Fig. 2(a) for three fixed values of the $\gamma_{2}$ parameter. One can see that functions $\gamma_{1 n}^{+}\left(\lambda, \gamma_{2}\right)$ decrease monotonically. Function graphs with different values of the parameter $\gamma_{2}$ do not intersect. A function graph with a larger parameter $\gamma_{2}$ value is below a function graph with a smaller parameter $\gamma_{2}$ value. This follows from the comparison of function values $\gamma_{1 n}^{+}=\gamma_{1 n}^{+}\left(\lambda, \gamma_{2}\right)$ in the middle of the $n$ interval at $\lambda_{n}^{*}=\nu_{n-1}+\pi / 4$. We have according to formula (18) $W\left(\lambda_{n}^{*}, \gamma_{2}\right)=0$, and we get the value of the function $\gamma_{1 n}^{+}\left(\lambda_{n}^{*}, \gamma_{2}\right)=1 /\left(\lambda_{n}^{*} \sqrt{\gamma_{2}}\right)$. Comparing two such values for two curves with different parameter values $\gamma_{2}^{(1)}>\gamma_{2}^{(2)}$ gives

$$
\frac{\gamma_{1 n}^{+}\left(\lambda_{n}^{*}, \gamma_{2}^{(1)}\right)}{\gamma_{1 n}^{+}\left(\lambda_{n}^{*}, \gamma_{2}^{(2)}\right)}=\sqrt{\frac{\gamma_{2}^{(2)}}{\gamma_{2}^{(1)}}}<1
$$

7. Functions $\gamma_{1 n}^{+}=\gamma_{1 n}^{+}\left(\lambda, \gamma_{2}\right)$ are monotonic and continuous in the domain of their definition $\lambda \in\left(\nu_{n-1}, \nu_{n}\right]$ at $n=1,2, \ldots$, and have inverse functions for a fixed parameter value $\gamma_{2}=\gamma_{2}^{(0)}$. We denote these inverse functions by $\lambda_{n}=\lambda_{n}^{+}\left(\gamma_{1}, \gamma_{2}\right)$. Functions $\lambda_{n}=\lambda_{n}^{+}\left(\gamma_{1}, \gamma_{2}\right)$ are monotonic, continuous in the domain of their $\gamma_{1}>0$ definition. The inverse function graphs $\lambda_{n}=\lambda_{n}^{+}\left(\gamma_{1}, \gamma_{2}\right)$ are shown in Fig. 2(b) for the same values of the $\gamma_{2}$ parameter. The graphs of these functions are symmetrical to the graphs of the functions $\gamma_{1 n}^{+}=\gamma_{1 n}^{+}\left(\lambda, \gamma_{2}\right)$ with respect to the bisector of the first quadrant.

Function graphs $\lambda_{n}=\lambda_{n}^{+}\left(\gamma_{1}, \gamma_{2}\right)$ for $n=1,2,3$ are graphs of the exact solution of the problem at fixed values of the $\gamma_{2}$ parameter. We find the sought values $\lambda_{n}$ of the Sturm-Liouville problem at the value of the parameter $\gamma_{1}=\gamma_{1}^{(0)}$ as ordinates of intersection points of the straight line (vertical straight line in Fig. 2(b)) with function graphs $\lambda=\lambda_{n}^{+}\left(\gamma_{1}, \gamma_{2}\right)$ at $n=1,2, \ldots$

\subsection{Approximation functional dependencies between eigenvalues and parameters of the Sturm-Liouville problem}

The result of the first step of the proposed method was obtaining functional dependencies $\gamma_{1}^{+}=\gamma_{1 n}^{+}\left(\lambda, \gamma_{2}\right)$ and graphs of the exact $\lambda_{n}=\lambda_{n}^{+}\left(\gamma_{1}, \gamma_{2}\right)$ solution. The disadvantage of the graphical solution of the characteristic equation is the difficulty of using its results in computer modeling of a physical problem.

In the second step, we approximate the functions $\gamma_{1}^{+}=\gamma_{1 n}^{+}\left(\lambda, \gamma_{2}\right)$ with elementary functions $\Gamma_{1 n}=\Gamma_{1 n}\left(\lambda, \gamma_{2}\right)$, where the first index 1 coincides with the index of the parameter $\gamma_{1}$, and the second index $n$ is the number of interval $\left(\nu_{n-1}, \nu_{n}\right]$ at which the functions are defined.

Let's choose functions $\Gamma_{1 n}\left(\lambda, \gamma_{2}\right)$ so that they have the properties 1-7 of the $\gamma_{1 n}^{+}\left(\lambda, \gamma_{2}\right)$ functions. Let interpolationapproximation functions $\Gamma_{1 n}\left(\lambda, \gamma_{2}\right)$ interpolate the values of the functions $\gamma_{1 n}^{+}=\gamma_{1 n}^{+}\left(\lambda, \gamma_{2}\right)$ at $\lambda=\nu_{n}$ and provide their limit values as $\lambda \rightarrow \nu_{n-1}+0$; let $\Gamma_{1 n}\left(\lambda, \gamma_{2}\right)$ approximate the values of the functions $\gamma_{1 n}^{+}=\gamma_{1 n}^{+}\left(\lambda, \gamma_{2}\right)$ at internal points of interval $\left(\nu_{n-1}, \nu_{n}\right)$ [25].

Taking into account properties 5 and $\mathbf{7}$ of the $\gamma_{1 n}^{+}=\gamma_{1 n}^{+}\left(\lambda, \gamma_{2}\right)$ functions, select functions $\Gamma_{1 n}=\Gamma_{1 n}\left(\lambda, \gamma_{2}\right)$ in the form of

$$
\gamma_{1 n}^{+} \approx \Gamma_{1 n}=\Gamma_{1 n}\left(\lambda, \gamma_{2}\right)=A_{n} \gamma_{2}^{s_{n}}\left(\frac{v_{n+1}^{2}-\lambda^{2}}{\lambda^{2}-v_{n}^{2}}\right)^{r_{n}}
$$

where parameters $A_{n}, s_{n}$ and $r_{n}$ are introduced. The additional condition $r_{n}>0$ provides the required function values at the ends of the $\left(\nu_{n-1}, \nu_{n}\right]$ interval. By verifying directly, one can find out that the functions selected in the form (20) satisfy the $\mathbf{1 - 4}$ properties. The additional condition $s_{n}<0$ ensures that property $\mathbf{6}$ is met. 

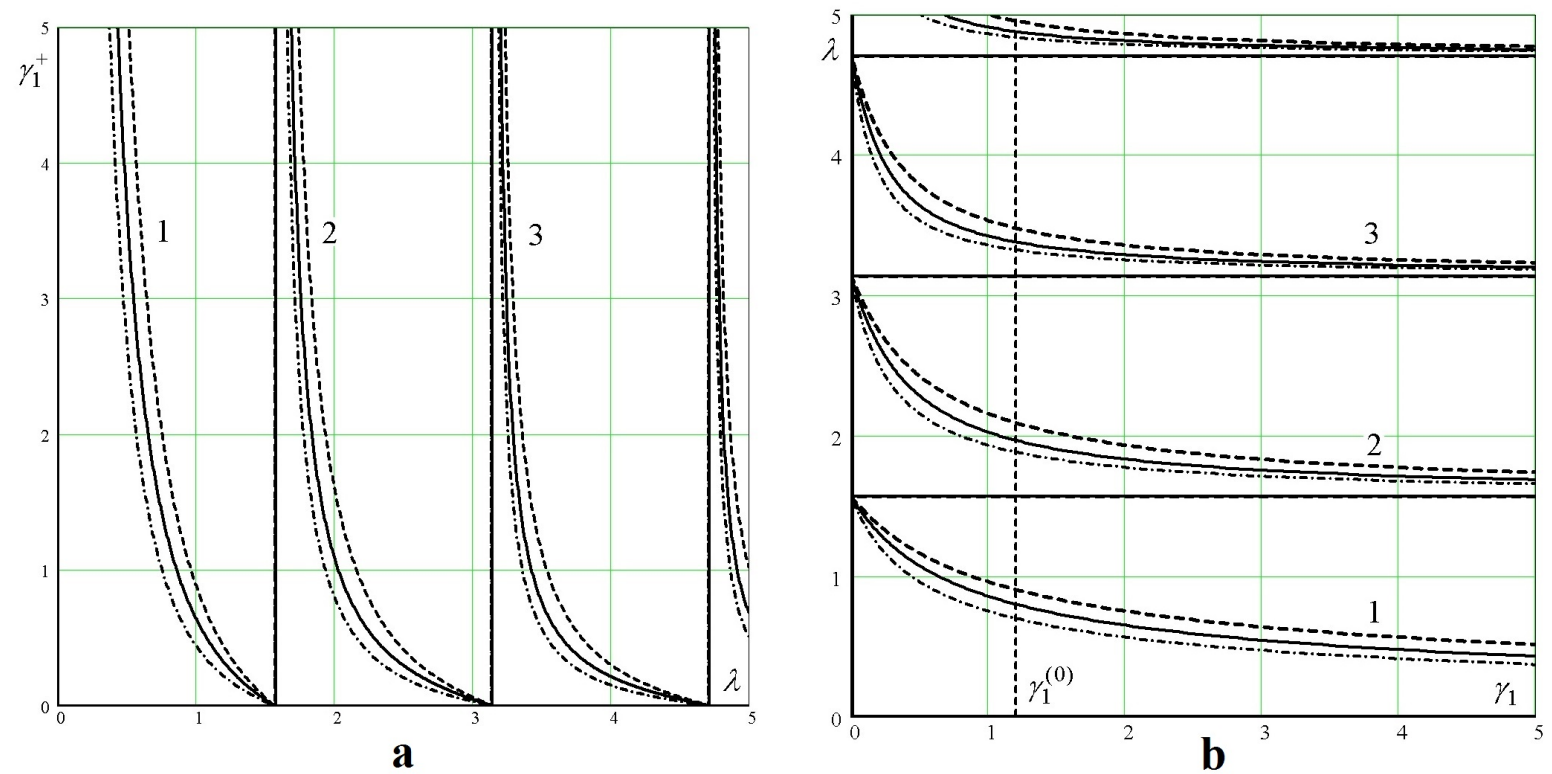

FIG. 2. Mutually inverse functions: $\gamma_{1}^{+}=\gamma_{1 n}^{+}\left(\lambda, \gamma_{2}\right)-(\mathrm{a}) ; \lambda=\lambda_{n}^{+}\left(\gamma_{1}, \gamma_{2}\right)-$ (b). Curves $1,2,3$ are constructed for functions with indices $n=1,2,3$ at three values of the $\gamma_{2}$ parameter: $\gamma_{2}=0.5$ - dashed lines; $\gamma_{2}=1.0$ - solid lines; $\gamma_{2}=2.0$ - chain-dotted lines

The parameters $A_{n}, s_{n}$ and $r_{n}$ introduced in the right side of formula (20) allow one to approximate the functions $\gamma_{1 n}^{+}=\gamma_{1 n}^{+}\left(\lambda, \gamma_{2}\right)$ at the internal points of the $\left(\nu_{n-1}, \nu_{n}\right]$ interval using the least squares method.

After calculating parameters, we get elementary functions $\Gamma_{1 n}=\Gamma_{1 n}\left(\lambda, \gamma_{2}\right)$.

Further consideration is carried out for an arbitrary fixed number $n$ of the $\left(\nu_{n-1}, \nu_{n}\right]$ interval.

The function $\Gamma_{1 n}=\Gamma_{1 n}\left(\lambda, \gamma_{2}\right)$ has the inverse function at interval $\left(\nu_{n-1}, \nu_{n}\right]$, and the inverse function is also elementary:

$$
\lambda_{n} \approx \hat{\lambda}_{n}=\sqrt{\frac{v_{n+1}\left(A_{n} \gamma_{2}^{s_{n}}\right)^{q_{n}}+v_{n+1}\left(\Gamma_{1 n}\right)^{q_{n+1}}}{\left(A_{n} \gamma_{2}^{s_{n}}\right)^{q_{n}}+\left(\Gamma_{1 n}\right)^{q_{n}}}}
$$

where $q_{n}=1 / r_{n}$.

Note that when solving the single-parameter problem in [22], when choosing the form of approximation $\Gamma=$ $\Gamma_{n}(\lambda)$ function, evenness of the function $\gamma_{n}^{+}(\lambda)$ relative to variable $\lambda$ was not taken into account. Accounting for the evenness of the function $\gamma_{1 n}^{+}\left(\lambda, \gamma_{2}\right)$ relative to variable $\lambda$ in formula (20) increases the approximation's accuracy.

We find the approximation parameters $r_{n}, s_{n}$ and $A_{n}$ by the least squares method, which provides the best approximation of the $\gamma_{1 n}^{+}=\gamma_{1 n}^{+}\left(\lambda, \gamma_{2}\right)$ function.

After calculating approximation parameters, the values for $\lambda_{n}$ are obtained using formula (21) at the given values of the problem parameters $\gamma_{1}$ and $\gamma_{2}$. Then, we find the eigenvalue $\mu_{n}=\lambda_{n}^{2}$ of the Sturm-Liouville problem.

\subsection{Finding of approximation parameters}

To find approximation parameters $A_{n}, s_{n}$ and $r_{n}$ we set $J$ of values for the problem parameter $\gamma_{2}: \gamma_{2}^{(j)}$, where $j=1,2, \ldots, J$. We will also select $M_{n}$ of values for the argument $\lambda_{n m}$, where $m=1,2, \ldots, M_{n}$ on each interval $\left(\nu_{n-1}, \nu_{n}\right)$. The values of the arguments $\gamma_{2}^{(j)} \in(0, T)$ and $\lambda_{n m} \in\left(\nu_{n-1}, \nu_{n}\right]$ must cover uniformly the domain of the function $\gamma_{1 n}^{+}=\gamma_{1 n}^{+}\left(\lambda, \gamma_{2}\right)$ definition, the quantity $T$ being determined by the largest value the parameter $\gamma_{2}$ can take.

Let's calculate $M J$ of values of the $\gamma_{1 n}^{+}=\gamma_{1 n}^{+}\left(\lambda, \gamma_{2}\right)$ function: $\gamma_{1 n m j}=\gamma_{1 n}^{+}\left(\lambda_{n m}, \gamma_{2}^{(j)}\right)$.

To apply the least squares method, take the logarithm of both sides of equation (20)

$$
\ln \left(\Gamma_{1 n}\left(\lambda, \gamma_{2}\right)\right)=q_{n}+s_{n} \ln \gamma_{2}+r_{n} R_{n}(\lambda),
$$

where

$$
\begin{gathered}
q_{n}=\ln \left(A_{n}\right), \\
R_{n}(\lambda)=\ln \left(\frac{v_{n+1}^{2}-\lambda^{2}}{\lambda^{2}-v_{n}^{2}}\right) .
\end{gathered}
$$


We will look for the $q_{n}, s_{n}$ and $r_{n}$ values from the condition of ensuring the minimum value of the function $F_{n}\left(q_{n}, s_{n}, r_{n}\right)$. The function $F_{n}\left(q_{n}, s_{n}, r_{n}\right)$ is the average value of the sum of the difference squares for values $\ln \left(\gamma_{1 n m j}^{+}\right)$and $\ln \left(\Gamma_{1 n}\left(\lambda_{n m}, \gamma_{2}^{(j)}\right)\right)$ :

$$
\begin{gathered}
F_{n}\left(q_{n}, s_{n}, r_{n}\right)=\overline{\left(\ln \gamma_{1 n m j}^{+}-\ln \left(\Gamma_{1 n}\left(\lambda_{n m}, \gamma_{2}^{(j)}\right)\right)\right)^{2}}= \\
=\frac{1}{M_{n} J} \sum_{j=1}^{J} \sum_{m=1}^{M_{n}}\left(\ln \gamma_{1 n m j}^{+}-q_{n}-s_{n} \ln \gamma_{2}^{(j)}-r_{n} R_{n}\left(\lambda_{n m}\right)\right)^{2} .
\end{gathered}
$$

Here and elsewhere, we use the symbol of averaging to calculate the average value of some variable value $Z$ if $Z$ takes the $z_{11}, z_{12}, \ldots, z_{M J}$ values:

$$
\bar{Z}=\frac{1}{M J} \sum_{m=1}^{M} \sum_{j=1}^{J} z_{m j} .
$$

The condition of the minimum for function $F_{n}\left(q_{n}, s_{n}, r_{n}\right)$ is the equality to zero of its partial derivatives regarding the arguments $q_{n}, s_{n}$ and $r_{n}$. This condition results in a system of linear algebraic equations regarding the quantities $q_{n}, r_{n}$, and $s_{n}$

$$
\mathbf{A}_{n} \mathbf{X}_{n}=\mathbf{B}_{n}
$$

where the elements of the third order matrix are: $A_{11}=1, A_{12}=A_{21}=\overline{\ln \gamma_{2}}, A_{13}=A_{31}=\overline{R_{n}}, A_{22}=\overline{\left(\ln \gamma_{2}\right)^{2}}$, $A_{23}=A_{32}=\overline{R_{n} \ln \gamma_{2}}, A_{33}=\overline{\left(R_{n}\right)^{2}} ; \mathbf{X}_{n}=\left(q_{n}, s_{n}, r_{n}\right)^{T}$ is matrix column of the required values, sign $T$ means transposition of matrix;

is the free member column.

$$
\left.\mathbf{B}_{n}=\overline{\left(\ln \gamma_{1 n m j}^{+}\right.}, \overline{\ln \gamma_{1 n m j}^{+} \ln \gamma_{2}}, \overline{\ln \gamma_{1 n m j}^{+} R_{n}}\right)^{T}
$$

The solution of system (26) gives values

$$
\mathbf{X}_{n}=\mathbf{A}_{n}^{-1} \mathbf{B}_{n}
$$

We find the approximation parameter $A_{n}$ from the relation (23)

$$
A_{n}=e^{q_{n}}
$$

\subsection{Results of numerical calculations of eigen frequencies}

To calculate the values of the function $\Gamma_{1 n}=\Gamma_{1 n}\left(\lambda, \gamma_{2}\right)$ at each $n$ interval $\left(\nu_{n-1}, \nu_{n}\right], n=1,2, \ldots, N$, we select the values of the first argument as $\lambda_{n m}=\lambda_{n 0}+\Delta \lambda(m-1), m=1,2, \ldots, M$, where $\lambda_{n 0}$ is the initial value, and $\Delta \lambda$ is the step of changing the argument $\lambda$. We select the values of the second argument $\gamma_{2 j}=\gamma_{0}+\Delta \gamma(j-1)$, $j=1,2, \ldots, J$, where $\gamma_{0}$ is the initial value, and $\Delta \gamma$ is the step of changing the argument $\gamma_{2}$. It was accepted for calculations: $M=8, J=5, \lambda_{n 0}=\nu_{n}+0.001, \Delta \lambda=\nu_{1} /(M+1)-0.01, \gamma_{0}=0.5, \Delta \gamma=0.5$.

The results for calculation of the approximation constants $A_{n}$ and $r_{n}$ by formulas (27) and (28) for the first ten intervals $\left(\nu_{n-1}, \nu_{n}\right], n=1,2, \ldots, 10$ are shown by points in Figs. 3,4.

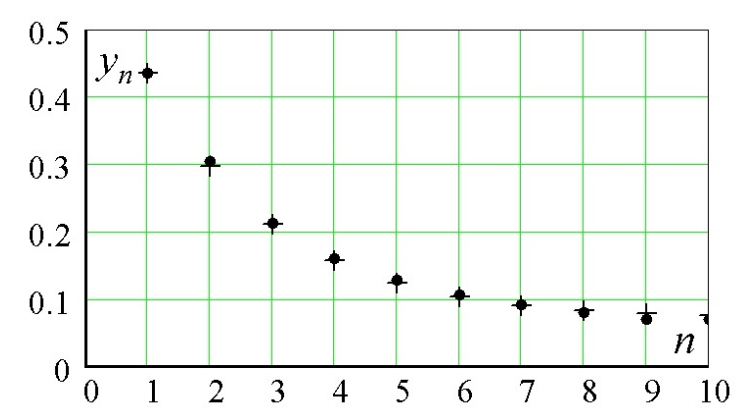

FIG. 3. Dependencies $y_{n}=A_{n}$ and $y_{n}=\hat{A}_{n}$ on interval number $n$ are shown by points and crosses respectively

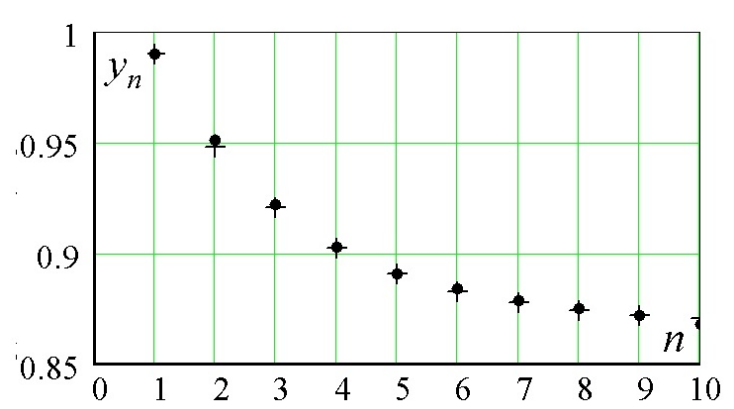

FIG. 4. Dependencies $y_{n}=r_{n}$ and $y_{n}=\hat{r}_{n}$ on interval number $n$ are shown by points and crosses respectively

Calculations showed also that the third approximation constant $s_{n}$ does not depend on the problem parameters $\gamma_{1}$ and $\gamma_{2}$. The value $s_{n}$ is equal to -0.5 for any $n$ with relative error not more than $0.1 \%$, which is in full compliance with the property 6 and the relation (19), of item 5.2. 
For numerical calculations of eigen frequency with any number $n$ according to formula (21), we use the found numerical values for parameters $A_{n}$ and $r_{n}$ at $n=1,2, \ldots, N$ to obtain their interpolation-approximation values $\hat{A}_{n}$ and $\hat{r}_{n}$ [25].

We find dependencies of the parameters $\hat{A}_{n}$ and $\hat{r}_{n}$ on interval number $n$ in the form:

$$
\begin{gathered}
\hat{A}_{n}=\varphi_{n}\left(p_{A}\right)=A_{N}+\left(A_{1}-A_{N}\right) e^{-p_{A}(n-1)} ; \\
\hat{r}_{n}=\psi_{n}\left(p_{r}\right)=r_{N}+\left(r_{1}-r_{N}\right) e^{-p_{r}(n-1)},
\end{gathered}
$$

where we distinguish interpolation nodes $n=1, n=N$ and approximation nodes $2 \leq n \leq N-1$. You have introduced parameters $p_{A}$ and $p_{r}$ to approximate dependencies.

We find the parameter $p_{A}$ so that approximate equalities $\hat{A}_{n} \approx A_{n}$ at $n=1,2, \ldots, N$ are performed in the best way.

According to equation (29) we have the exact equality $\hat{A}_{1}=A_{1}$ at $n=1$, and we have the approximate equality $\hat{A}_{N} \approx A_{N}$, where $N$ is large enough.

We find values $p_{n}$ providing the equalities $A_{n}=\varphi_{n}\left(p_{n}\right)$ for other values $n=2, \ldots, N-1$ :

$$
p_{n}=\varphi_{n}^{-1}\left(A_{n}\right)=\frac{1}{n-1} \ln \left(\frac{A_{1}-A_{N}}{A_{n}-A_{N}}\right) .
$$

Using the least squares method, we calculate the optimal value of the parameter $p_{A}$, which provides the minimum of the value $d$. This value is average squared displacement of the value $p_{A}$ from all calculated values $p_{n}$ at $n=$ $2, \ldots, N-1$

$$
d=d\left(p_{A}\right)=\frac{1}{N-2} \sum_{n=2}^{N-1}\left(p_{n}-p_{A}\right)^{2} .
$$

From the minimum condition of the function $d=d\left(p_{A}\right)$ we get the optimal value of the approximation parameter as the average value of all calculated $p_{n}$

$$
p_{A}=\frac{1}{N-2} \sum_{n=2}^{N-1} p_{n}
$$

where averaging is performed without taking into account edge values $A_{1}$ and $A_{N}$.

Similar reasoning for parameter $p_{r}$ leads to the formula

$$
p_{r}=\frac{1}{N-2} \sum_{n=2}^{N-1} \frac{1}{n-1} \ln \left(\frac{r_{1}-r_{10}}{r_{n}-r_{10}}\right) .
$$

The calculation of parameters $p_{A}$ and $p_{r}$ by formulas (31) and (32) using the data given in Figs. 3,4 gave values $p_{A}=0.48$ and $p_{r}=0.42$. Having obtained the optimal value of approximation parameters $p_{A}$ and $p_{r}$ we calculate values $\hat{A}_{n}$ and $\hat{r}_{n}$ for $n=1,2, \ldots, N$ by formulas (29) and (30).

The calculation results $\hat{A}_{n}$ and $\hat{r}_{n}$ at $\mathrm{N}=10$ are shown by crosses in Figs. 3,4.

Fig. 5 shows the graphs of the exact dependencies of the first two eigen frequencies $\lambda_{1}=\lambda_{1}\left(\gamma_{1}, \gamma_{2}\right)$ and $\lambda_{2}=$ $\lambda_{2}\left(\gamma_{1}, \gamma_{2}\right)$ on the parameter $\gamma_{1}$ for three fixed values of the parameter $\gamma_{2}$. These dependencies are represented by solid lines. The exact frequencies values were plotted using the technique shown in Fig. 2(b). The dependencies of approximate values of eigen frequencies $\hat{\lambda}_{1}=\hat{\lambda}_{1}\left(\gamma_{1}, \gamma_{2}\right)$ and $\hat{\lambda}_{2}=\hat{\lambda}_{2}\left(\gamma_{1}, \gamma_{2}\right)$ on the parameter $\gamma_{1}$ are shown in Fig. 5 by dashed lines. Approximate frequencies values were calculated using formulas (21), (31) and (32).

\subsection{About error of eigen frequencies calculation}

We calculate relative error of found eigen frequency value on $n$ interval for estimation of accuracy of approximate formula (21)

$$
\delta_{n}=\left|\frac{\lambda_{n}-\hat{\lambda}_{n}}{\lambda_{n}}\right|=\left|1-\frac{\hat{\lambda}_{n}}{\lambda_{n}}\right| .
$$

We have the previously obtained analytical dependence $\gamma_{1 n}^{+}=\gamma_{1 n}^{+}\left(\lambda, \gamma_{2}\right)$ given by formula (18). Approximation of this dependence $\Gamma_{1 n}=\Gamma_{1 n}\left(\lambda, \gamma_{2}\right)$ is introduced by formula (20). The function $\Gamma_{1 n}=\Gamma_{1 n}\left(\lambda, \gamma_{2}\right)$ has the inverse function $\hat{\lambda}_{n}=\Gamma_{1 n}^{-1}\left(\gamma_{1}, \gamma_{2}\right)$. 




FIG. 5. Dependencies of exact eigen frequencies $\lambda_{1}$ and $\lambda_{2}$ (solid curves 1,2) and approximate eigen frequencies $\hat{\lambda}_{1}$ and $\hat{\lambda}_{2}$ (dashed lines 1,2) on parameter $\gamma_{1}$ at three fixed values of parameter $\gamma_{2}$ : curves (a) - for $\gamma_{2}=2,0$; curves (b) - for $\gamma_{2}=1,0$; curves (c) - for $\gamma_{2}=0,5$

We get a parametric functional dependence $\delta_{n}=\delta_{n}\left(\gamma_{1}, \gamma_{2}\right)$ if we consider the quantity $\lambda$ as a parameter. Indeed, if the value $\lambda_{n}$ is set, we have the following values: $\gamma_{1 n}^{+}\left(\lambda_{n}\right)=\gamma_{1 n}^{+}\left(\lambda_{n}, \gamma_{2}\right)$ and $\hat{\lambda}_{n}=\Gamma_{1 n}^{-1}\left(\gamma_{1}^{+}, \gamma_{2}\right)=$ $\Gamma_{1 n}^{-1}\left(\gamma_{1 n}^{+}\left(\lambda_{n}, \gamma_{2}\right), \gamma_{2}\right)$. Then, taking into account equality (33), we give the function $\delta_{n}=\delta_{n}\left(\gamma_{1}, \gamma_{2}\right)$ parametrically:

$$
\left\{\begin{array}{l}
\delta_{n}\left(\lambda_{n}\right)=\left|1-\frac{1}{\lambda_{n}} \Gamma_{1 n}^{-1}\left(\gamma_{1 n}^{+}\left(\lambda_{n}, \gamma_{2}\right), \gamma_{2}\right)\right| \\
\gamma_{1 n}^{+}\left(\lambda_{n}\right)=\gamma_{1 n}^{+}\left(\lambda_{n}, \gamma_{2}\right)
\end{array}\right.
$$

Relative errors of eigen frequency calculations at the first ten intervals were obtained by formulas (34) for case $\gamma_{2}=1$.

The table shows estimates $\Delta_{n}$ of the largest values of relative errors at the first ten intervals: $\delta_{n} \leq \Delta_{n}$ at $\lambda \in\left(\nu_{n-1}, \nu_{n}\right]$.

TABLE 1. Estimates $\Delta_{n}$ of relative error of eigen frequency calculations on intervals with number $n$

\begin{tabular}{|c|c|c|c|c|c|c|c|c|c|c|}
\hline$n$ & 1 & 2 & 3 & 4 & 5 & 6 & 7 & 8 & 9 & 10 \\
\hline$\Delta_{n}$ & $0.01 \%$ & $1.0 \%$ & $0.9 \%$ & $0.5 \%$ & $0.3 \%$ & $0.2 \%$ & $0.1 \%$ & $0.1 \%$ & $0.05 \%$ & $0.01 \%$ \\
\hline
\end{tabular}

It can be seen from the table that relative errors of eigen frequency calculations, starting from the third, monotonically decrease.

\section{Conclusion}

The presence of approximate analytical dependencies for the eigenvalues of the Sturm-Liouville problem on the problem parameters makes it possible to carry out a comprehensive mathematical study of the physical phenomenon from which the Sturm-Liouville problem is derived.

For example, it may be recommended to use the proposed method of solving characteristic equations to solve inverse problems, such as identification of boundary conditions of spectral problems by eigenvalues [26], identification of local defects in mechanical objects (rods, beams, pipelines) [27].

Use of the proposed method in solving inverse problems of oscillations of micro- and nano-objects, in particular MEMS and NEMS, will allow expanding the possibilities of nanometrology. 


\section{References}

[1] Margolin V.I. Zhabrev V.A., Lukyanov G.N., Tupik V.A. Introduction to Nanotechnologies. Lan', Saint Petersburg, 2012 , 464 p.

[2] Voitovich I.D., Korsunsky V.M. Intellectual sensors. BINOMIAL. Knowledge Laboratory, M., 2012, 624 p.

[3] Nguyen C.T.-C. MEMS technology for timing and frequency control. IEEE Trans. Ultrason. Ferroelectr. Freq. Control, 2007, 54, P. 251-270.

[4] Basu J., Bhattacharyya T.K. Microelectromechanical resonators for radio frequency communication applications. Microsyst. Technol., 2011, 17, P. 1557-1580.

[5] NEMS/MEMS Technology and Devices. Edited by Lynn Khine and Julius M. Tsai. International Conference on Materials for Advanced Technologies (ICMAT 2011), Symposium G: NEMS/MEMS and microTAS, June 26-July 1, Suntec, Singapore, 2011,242 p.

[6] Greenberg Y.S., Paskin Yu.A., Ilichev K.V. Nanomechanical resonators. Successes of Physical Sciences, 2012, 4(182), P. 408-436.

[7] Mukhurov N.I., Efremov G.I. Electromechanical micro-devices. Belarusian. Navuka, Minsk, 2012, 257 p.

[8] Van Beek, J.T.M., Puers, R. A review of MEMS oscillators for frequency reference and timing applications. J. Micromech. Microeng., 2012, 22, P. 13001.

[9] Nguyen, C.T.-C. MEMS-based RF channel selection for true software-defined cognitive radio and low-power sensor communications. IEEE Commun. Mag., 2013, 51, P. 110-119.

[10] Uranga A., Verd J., Barniol N. CMOS-MEMS resonators: From devices to applications. Microelectron. Eng., 2015,132, P. 58-73.

[11] Abdolvand R., Bahreyni B., Lee J., \& Nabki F. Micromachined Resonators: A Review. Micromachines, 2016, 7(9), 2016, P. 160-213.

[12] Ali W.R., Prasad M. Piezoelectric MEMS based acoustic sensors: A review. Sensors and Actuators A: Physical. Sensors and Actuators A., 2020, 301, P. 2-31.

[13] Lyshevski S.E. MEMS and NEMS Systems, Devices, and Structures. CRC Press, New York, 2002, 461 p.

[14] Kolmakov A.G., Barinov S.M., Alymov M.I. Basis of technologies and application of nanomaterials. Fizmatlitis., M.,2012, 208 p.

[15] Zaytsev V.F., Polyanin A.D. Handbook on Ordinary Differential Equations. Fizmatlit, M., 2001, 576 p.

[16] Collatz L. Tasks for own values (with technical annexes). Science, M., 1968, 504 pp.

[17] Vibrations in engineering: Reference. T. 1. Fluctuations of linear systems. Under Ed. V.V. Bolotina. Mashinostroenie, M., 1978, 352 p.

[18] harles T. Fulton Two-point boundary value problems with eigenvalue parameter contained in the boundary conditions. Proceedings of the Royal Society of Edinburgh, 1977, 77 A, P. 293-308.

[19] Shkalikov A.A. Boundary problems for ordinary differential equations with parameter under boundary conditions. Works of I.G. Petrovsky Seminar, 1983, 9, P. 190-229.

[20] Titchmarsh E.C. Eigenfunction expansions associated with second order differential equations. V. 1. Oxford Univ. Press, London, 1962, 203 p.

[21] Naimark M.A. Linear differential operators. Science, M., 1969, 528 p.

[22] Lukyanov V.D., Nosova L.V., Bogorodsky A.V. et al. Approximate solution to the Sturm-Liuville problem with complicated boundary conditions. Sea intellectual technologies, 2019, 1(43), P. 142-146.

[23] Beckman I.N. Mathematics of Diffusion. OntoPrint, M., 2016, 400 p.

[24] Koshlyakov N.S., Gliner E.B., Smyrnov M. M. Differential equations of mathematical physics. Fizmatgiz, M., 1962,768 p.

[25] Lukyanov V.D. On the construction of an interpolation-approximation polynomial. Nanosystems: physics, chemistry, mathematics. 2012, 3(6), P. 5-15.

[26] Akhtyamov A.M. Theory of identification of edge conditions and its application. Fizmatlit, M., 2009, 272 p.

[27] Akhtyamov A. M., Ilgamov M. A. Review of research on identification of local defects of rods. Problems of machine building and reliability, 2020, 2, P. 3-15. 